\title{
Inflammatory endobronchial polyposis with bronchiectasis in cystic fibrosis
}

\author{
C. Roberts*, A.M. Devenny", R. Brooker\#, J.S. Cockburn", K.M. Kerr*
}

\begin{abstract}
Inflammatory endobronchial polyposis with bronchiectasis in cystic fibrosis. C. Roberts, A.M. Devenny, R. Brooker, J.S. Cockburn, K. M. Kerr. C) ERS Journals Ltd 2001.

ABSTRACT: An unusual case of endobronchial polyposis associated with extensive bronchiectasis in the context of cystic fibrosis (CF) has been described.

A 15-yr-old female patient with $C F$ underwent partial pneumonectomy for extensive bronchiectasis and frequent infective pulmonary exacerbations.

Cylindrical bronchiectasis with associated purulent bronchitis and bronchiolitis, together with inflammatory polyposis, was noted in the resected lung.

To the best of the authors' knowledge, this is the first report of multiple endobronchial polyposis and may represent a rare complication of bronchiectasis in a patient with cystic fibrosis. On-going infection and the cellular composition of the polyps are discussed in relation to their possible aetiological relevance and relationship to upper respiratory tract polyps.
\end{abstract}

Eur Respir J 2001; 18: 612-615.

Inflammatory bronchial polyps are rare lesions that are almost universally solitary and apparently occur spontaneously, or after airway injury. Multiple polyps are exceedingly rare in any circumstance. Bronchiectasis and inflammatory changes in airways are well recognized in cystic fibrosis $(\mathrm{CF})$. This report describes the clinical and pathological findings in a unique case of a patient with $\mathrm{CF}$ complicated by bronchiectasis, who developed widespread inflammatory polyps (polyposis) within bronchiectatic airways.

\section{Case report}

A 6-week-old female infant was first admitted to hospital with fever, nasal snuffles and poor feeding. Examination revealed bronchial breathing over the right chest, and right upper zone consolidation was confirmed by radiography. Treatment was started with antibiotics. Culture of upper airway secretions yielded Streptococcus pneumoniae, Staphylococcus aureus and Pseudomonas aeruginosa. Following good recovery the patient was discharged on prophylactic flucloxacillin. A repeat chest radiograph showed residual opacification, only of the right mid-zone.

Two weeks later the patient was readmitted with persistant cough and failure to thrive. Repeat chest radiography showed recurrent right upper zone consolidation. Further treatment with intravenous antibiotics was given and the patient was started on Progestimil with calorie supplements. Subsequent sweat tests showed osmolalities of 247 and

\begin{abstract}
*Dept of Pathology, Aberdeen Royal Infirmary, "Dept of Medical Paediatrics, Royal Aberdeen Children's Hospital and Aberdeen Royal Infirmary, Aberdeen, UK.
\end{abstract}

Correspondence: K.M. Kerr, Dept of Pathology, Aberdeen Royal Infirmary, Foresterhill, Aberdeen AB25 2ZD, UK. Fax: 441224663002

Keywords: Bronchiectasis cystic fibrosis

polyp

polyposis

Received: January 182001

Accepted after revision March 132001
$347 \mathrm{mosmol} \cdot \mathrm{kg}^{-1}$ (normal range $62-137 \mathrm{mosmol} \cdot \mathrm{kg}^{-1}$ ) confirming a diagnosis of CF. The patient was commenced on regular chest physiotherapy and Pancrex $\mathrm{V}$. The patient's CF genotype was later identified as G551D/E60X.

Thereafter, the patient made good progress and required approximately four courses of oral antibiotics per year for chest infections. A cough swab taken at age 3 yrs yielded $S$. aureus, but regular sputum samples were not available until the patient was $5 \mathrm{yrs}$ of age. These revealed that the patient's respiratory tract was colonized with $P$. aeruginosa. At this stage, the patient's lung function was good (forced expiratory volume in one second (FEV1) $\sim 80 \%$, forced vital capacity (FVC) $\sim 90 \%$ predicted) and nebulized gentamicin was commenced. However, over the next 12 months the patient had poor weight gain and a reduction in lung function (FEV1 63\%, FVC $72 \%$ pred). After courses of both oral and intravenous antipseudomonal therapy, the patient was commenced on nebulized piperacillin. Coinciding with this, there was an improvement in growth and lung function.

From midchildhood, the patient was troubled with severe frontal headaches thought to be due to chronic sinusitis. Cranial magnetic resonance imaging (MRI) showed evidence of polypoidal disease involving both antra, the ethmoid complex, and extending into the frontal and sphenoidal sinuses. However, the patient never had any evidence of nasal polyps and responded well to intranasal corticosteroids.

From 12 yrs of age the patient began to have more 
frequent chest infections, often associated with minor chest pain and haemoptysis. Sputum isolates of $P$. aeruginosa were becoming increasingly resistant to oral antibiotics. In view of the patient's recurrent chest infections, computed tomography (CT) of the thorax was performed, indicating widespread bronchiectasis, the worst affected area being the right upper lobe with mediastinal shift (fig. 1). It was decided to proceed to partial pneumonectomy to improve the patient's quality of life. At surgery, the horizontal fissure was obliterated by adhesions, thereby necessitating resection of the right upper and middle lobes.

The postoperative period was complicated by a persistant air-leak, but following this, the patient made good progress with a significant reduction in the frequency of chest infections. The patient's lung function (postoperative FEV1 44\% pred) improved $\sim 7$ months later ( $52 \%$ pred).

\section{Pathological findings}

The right upper lobe revealed extensive cylindrical bronchiectasis in the medium- and small-sized bronchi, together with multiple endobronchial polyps measuring up to $4 \mathrm{~mm}$ throughout the bronchi and bronchioles of the upper lobe, filling the bronchiectatic airways (fig. 2).

Microscopic examination showed abnormally large airways, in which the normal elastic and muscular components of the bronchial walls had been replaced by a fibrovascular granulation tissue containing chronic inflammatory cells. The overlying columnar epithelium was pseudostratified and ciliated in places. Adjacent bronchi and bronchioli contained pus. The polyps were formed by a cellular, chronically inflamed fibro-connective tissue stroma, lined by a stratified columnar ciliated epithelium.

The cells in the stroma were mostly lymphocytes

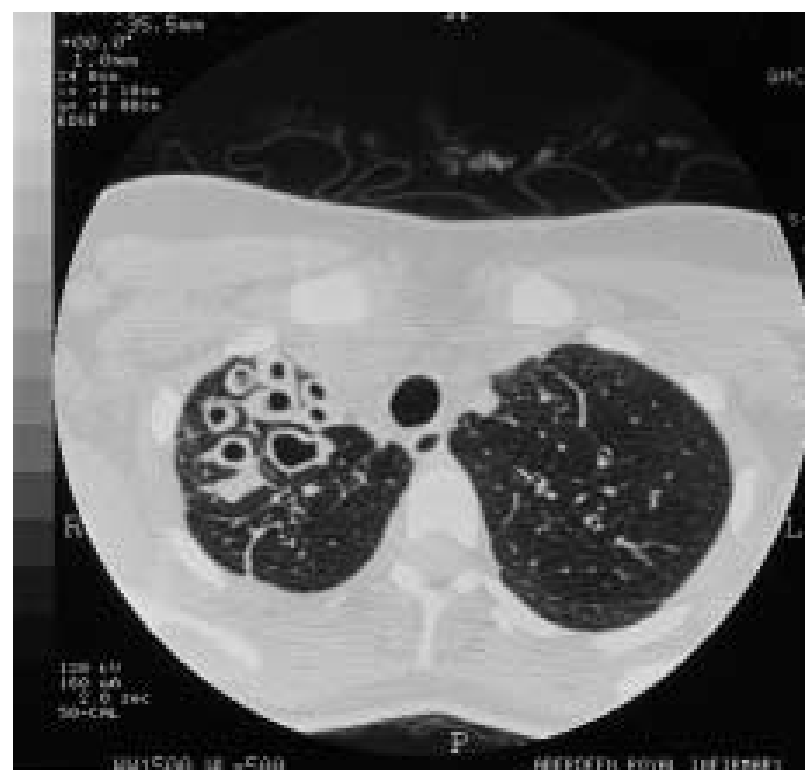

Fig. 1.-Computed tomography of the thorax showing right upper lobe ectatic bronchi with thickened walls.

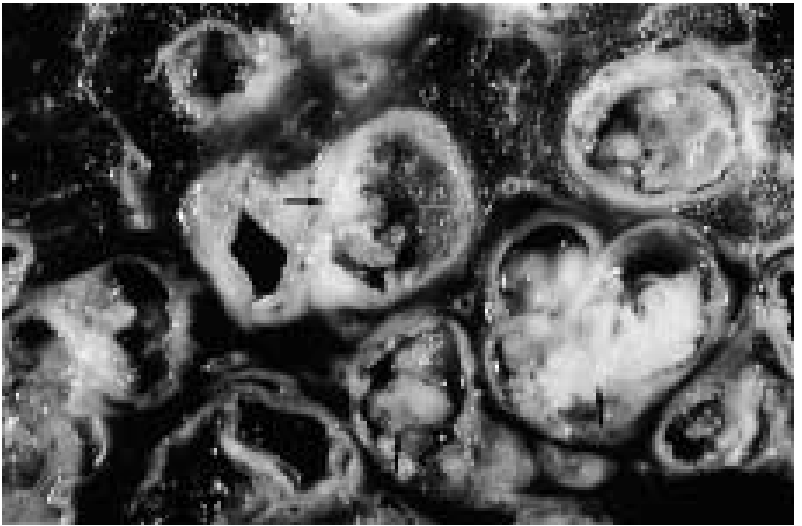

Fig. 2.-Gross appearance of lobectomy specimen. Bronchiectatic airways show multiple polyps partly filling airspaces, mucopus is also present.

and plasma cells, with a varied number of eosinophils and occasional mast cells. Neutrophil polymorphs were not part of the inflammatory cell infiltrate. Squamous metaplasia was not identified (fig. 3).

A morphological diagnosis of cylindrical bronchiectasis with associated purulent bronchitis and bronchiolitis, and inflammatory endobronchial polyposis of the upper lobe was made.
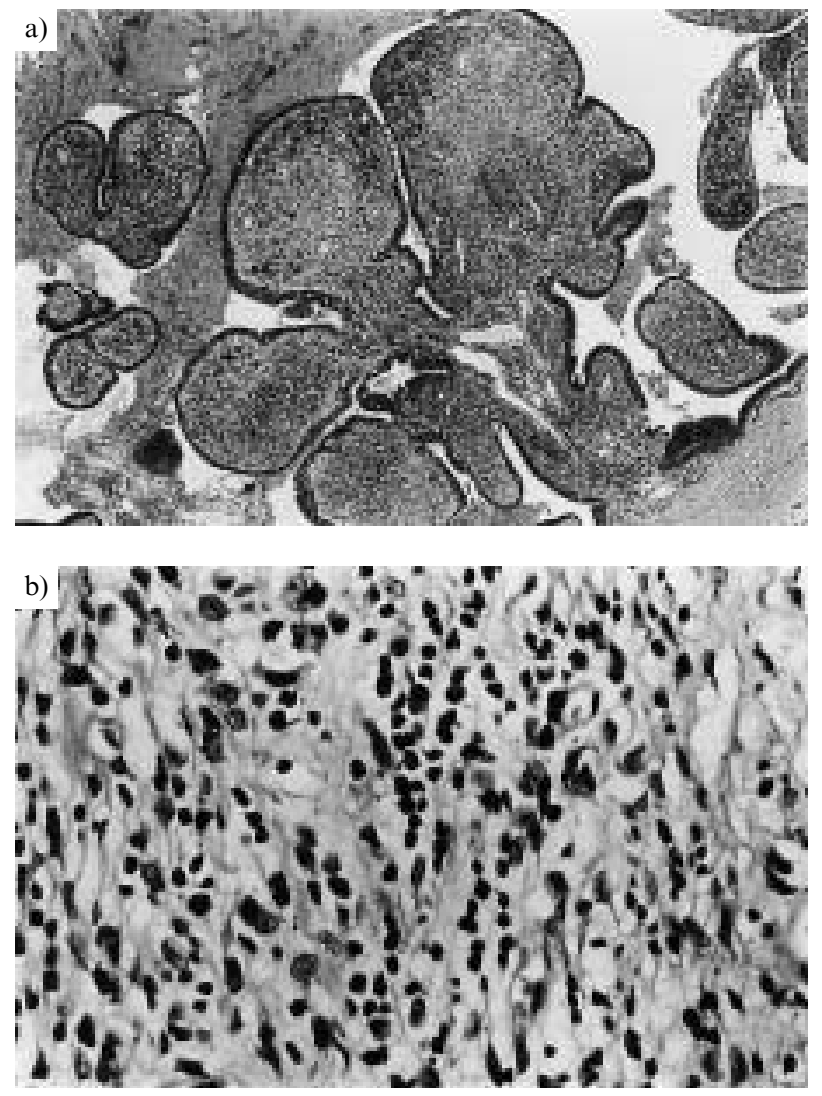

Fig. 3.-a) Photomicrograph of endobronchial polyposis. Polyps have complex branched architecture, are covered by respiratory epithelium and have an inflamed fibrovascular core. b) Photomicrograph showing lymphocytes and plasma cells in the inflammatory infiltrate. Note the absence of neutrophils. 


\section{Discussion}

Cases of bronchial polyps of inflammatory origin (inflammatory endobronchial polyps) have occasionally been described in the literature. The overwhelming majority of cases reported to date describe solitary lesions that apparently occurred in a major bronchus without associated tracheobronchial or pulmonary disease $[1,2]$, sometimes in relation to retained endobronchial foreign bodies [3, 4] or previous surgical treatment [5]. Removal of the foreign body or inhalation of corticosteroids appear to be successful therapeutic options. There are also case reports of solitary endobronchial inflammatory polyps that are associated with pulmonary disease, e.g. asthma [6] or chronic smoke injury [7]. One case has been reported [2] that exhibited multiple lesions.

The patient reported here was diagnosed with $\mathrm{CF}$ when aged 2 months. The patient showed rhinosinusitis, although nasal polyps did not develop, and later developed radiological evidence of bronchiectasis in the right upper lobe.

Bronchiectasis always complicates CF [8], although its distribution within the lungs is discussed controversially in the literature. DAIL and HAMMER [8] describe the occurrence of bronchiectasis as equally frequent in the upper and lower lobes, whereas THOMASHEFsKi et al. [9] found, in a necropsy study, that the upper lobes are generally more severely affected. In the present case, bronchiectasis occurred predominantly in the upper lobe. These bronchiectatic airways contained multiple endobronchial polyps. This is an extremely unusual finding and, to the authors' knowledge, has not been reported to date, although one report describes multiple polyps comprising "inflammatory tissue" in the right main bronchus in a patient who had had influenza 8 yrs previously [2]. Apart from the different previous respiratory history, it is difficult to conclude any more about this case since no histological details are given.

The aetiology of solitary inflammatory endobronchial polyps is thought to relate to the stagnation of purulent secretion within bronchi as the primary and perpetuating effect leading to bronchial mucosal hyperplasia and polyp formation $[10,11]$. Further studies $[2,12,13]$ provide detailed interpretation of the aetiology of solitary endobronchial polyps in the context of a clinical history of bronchial inflammation. The authors postulate a break in the mucosa following infection, leading to the formation of granulation tissue and deposition of fibrous tissue with subsequent epithelialization by pseudostratified, low-cuboidal or squamous epithelium, as well as proliferation of fibrous and epithelioid elements. In the present case, it might be assumed that both suggested aetiological mechanisms (stagnation of purulent secretion and ulceration of the mucosa) may have played a role in the genesis of the multiple endobronchial polyps. However, as fully-fledged endobronchial inflammatory polyposis is clearly unusual, with no descriptions in the literature, particularly in association with $\mathrm{CF}$, the above postulated aetiological processes for the genesis of solitary endobronchial polyps or multiple lesions may not be the complete story. However, the sustained inflammation, which is characteristic of CF [14], may be related to this rare pattern of mucosal reaction.

Approximately $20-32 \%$ of patients with CF develop nasal polyposis [11], the presence of which is apparently unrelated to the severity of the lung disease [11]. Nasal polyps that arise in the background of $\mathrm{CF}$ have been described as neutrophilic rather than eosinophilic $[15,16]$. Nasal polyps that occur without the context of CF frequently contain large numbers of eosinophils, pointing to a possible allergic aetiology $[15,16]$. While the endobronchial polyps in the present case did not have the neutrophils frequently described in nasal polyps of $\mathrm{CF}$ patients, the polyposis present in the patient's bronchiectatic airways lacked the large number of eosinophils and oedematous stroma that characterizes the allergic nasal polyp. The authors, therefore, think an allergic aetiology for the endobronchial polyposis unlikely. It is, however, possible that once established, the endobronchial polyposis contributed to obstruction and poor drainage of the bronchiectatic airway, making recurrent infection more likely and exacerbating the chronic inflammatory process. This, in turn, may have promoted polyp formation. The patient had sinus polyposis demonstrated radiologically but these were never biopsied.

The main differential diagnosis of solitary inflammatory endobronchial polyp is glandular bronchial papilloma. This is a rare solitary endobronchial neoplasm that has a distinct morphologic appearance, i.e. a relatively noninflamed papillary stromal core that is lined by a pseudostratified or a single layer of ciliated or nonciliated columnar cells, admixed with cuboidal or mucin-filled cells [17]. The endobronchial polyps in the present case were not only morphologically different from a papilloma, but were also multiple, coating the abnormal luminal surface.

In summary, multiple endobronchial polyposis could represent a very rare, late complication of cystic fibrosis associated with bronchiectasis. The limitation of the polyps to the upper lobe suggests that local factors (inflammation, stagnation of purulent secretion) may be involved in the aetiology of these lesions.

\section{References}

1. Tedeschi LG, Libertini R, Conte B. Endobronchial polyp. Chest 1973; 63: 110-112.

2. Jackson C, Jackson CL. Benign tumors of the trachea and bronchi, with special references to tumor-like formation of inflammatory origin. JAMA 1932; 99: $1747-1754$.

3. Moisan TC. Retained endobronchial foreign body removal facilitated by steroid therapy of an obstructing inflammatory polyp. Chest 1991; 100: 270.

4. Berman DE, Wright ES, Edstrom HW. Endobronchial inflammatory polyp associated with a foreign body. Successful treatment with corticosteroids. Chest 1984; 86: 483-484. 
5. Ikeda M, Ishida H, Tsujimoto S, Kato H. Endobronchial inflammatory polyp after thoracoabdominal aneurysm surgery: a late complication of use of a double-lumen endobronchial tube. Anesthesiology. 1996; 84: 1234-1236.

6. Niimi A, Amitani R, Ikeda T, Kubo Y, Tanaka E, Kuze F. Inflammatory bronchial polyps associated with asthma: resolution with inhaled corticosteroid. Eur Respir J 1995; 8: 1237-1239.

7. Smith RE. Endobronchial polyp and smoke injury. Postgrad Med J 1989; 65: 785-787.

8. Dail DH. Bronchial and Transbronchial Diseases. In: Dail DH, Hammer SP, eds. Pulmonary Pathology, 2nd edn. Springer Verlag, 1993; pp. 102-103.

9. Thomashefski JF, Bruce M, Stern RC, Dearborn DG, Dahms B. Pulmonary air cysts in cystic fibrosis: relation of pathologic features to radiologic findings and history of pneumothorax. Human Pathol 1985; 16: 253-261.

10. Rosenberg JC. Bronchial polyps of inflammatory origin. J Thorac Cardiovasc Surg 1969; 57: 848-852.

11. Greening AP. Cystic Fibrosis. In: Seaton A, Seaton D,
Leitch AG, eds. Crofton and Douglas' Respiratory Diseases, Vol. 2, 5th edn. Blackwell Science, 2000; pp. 853-856.

12. Jackson C, Jackson CL. Benign tumors and tumorlike conditions in the tracheobronchial tree. Am J Surg 1938; 42: 275-281.

13. Freant LJ, Sawyers JL. Benign bronchial polyps and papillomas. Ann Thorac Surg 1971; 11: 460-467.

14. Konstan MW, Berger M. Current understanding of the inflammatory process in cystic fibrosis: onset and etiology. Pediatr Pulmonol 1997; 24: 137-142.

15. Rowe-Jones JM, Trendell-Smith N, Mackay IS. Polypoidal rhinosinusitis in cystic fibrosis: a clinical and histopathological study. Clin Otolaryngology \& Allied Sciences 1997; 22: 167-171.

16. Rowe-Jones JM, Trendell-Smith N, Shembekar M, Mackay IS. Polypoid rhinosinusitis in patients with host defence deficiencies: cellular infiltration and disease severity. Rhinology 1997; 35: 113-117.

17. Flieder DB, Koss MN, Nicholson A, et al. Solitary pulmonary papillomas in adults. Am J Surg Pathol 1998; 22: 1328-1342. 